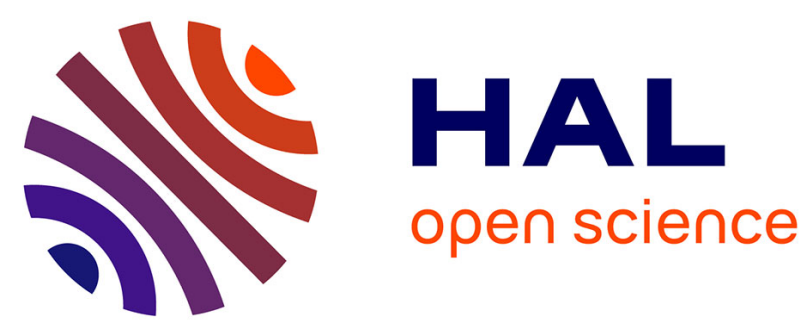

\title{
Implantation d'oxygène et co-implantation d'oxygène et de silicium, à faible dose, dans GaAs. II. Etude des défauts profonds
}

\author{
A. Le Bloa, Dang Tran Quan, Z. Guennouni, P.N. Favennec
}

\section{- To cite this version:}

A. Le Bloa, Dang Tran Quan, Z. Guennouni, P.N. Favennec. Implantation d'oxygène et coimplantation d'oxygène et de silicium, à faible dose, dans GaAs. II. Etude des défauts profonds. Revue de Physique Appliquée, 1989, 24 (10), pp.983-991. 10.1051/rphysap:019890024010098300 . jpa-00246136

\section{HAL Id: jpa-00246136 https://hal.science/jpa-00246136}

Submitted on 1 Jan 1989

HAL is a multi-disciplinary open access archive for the deposit and dissemination of scientific research documents, whether they are published or not. The documents may come from teaching and research institutions in France or abroad, or from public or private research centers.
L'archive ouverte pluridisciplinaire HAL, est destinée au dépôt et à la diffusion de documents scientifiques de niveau recherche, publiés ou non, émanant des établissements d'enseignement et de recherche français ou étrangers, des laboratoires publics ou privés. 


\title{
Implantation d'oxygène et co-implantation d'oxygène et de silicium, à faible dose, dans GaAs. II. Etude des défauts profonds
}

\author{
A. Le Bloa $\left({ }^{1}\right)$, Dang Tran Quan $\left({ }^{1}\right)$, Z. Guennouni $\left({ }^{1}\right)$ et P. N. Favennec $\left({ }^{2}\right)$ \\ (1) Groupe de Physique Cristalline, UA 804 CNRS, Université de Rennes I, Campus de Beaulieu, \\ 35042 Rennes Cedex, France \\ ( $\left.{ }^{2}\right)$ Groupement ICM/TOH, Centre National d'Etudes des Télécommunications, Lannion B, 22301 Lannion \\ Cedex, France
}

(Reçu le 23 novembre 1988, révisé le 15 juin 1989, accepté le 30 juin 1989)

\begin{abstract}
Résumé. - Nous présentons les résultats de l'étude, par la méthode DDLTS, des centres profonds dans les échantillons de GaAs implantés ou co-implantés, à faible dose, en oxygène ou en silicium et oxygène. Trois groupes de centres profonds ont été mis en évidence dans le domaine de températures 100-300 K. Les énergies d'activation du taux d'émission de ces centres sont comprises entre 0,1 et $0,6 \mathrm{eV}$. Ces centres profonds sont liés soit aux désordres du réseau cristallin du substrat, soit aux complexes formés par les ions implantés et les imperfections de ce réseau.

Abstract. - DDLTS results for oxygen low dose implanted GaAs and silicon-oxygen low dose coimplanted GaAs are presented. Three deep level groups are detected in 100-300 K. Activation energy was measured between 0.1-0.6 eV. These deep levels are in relation with crystallizing disorder of GaAs substrate and with complexes formed by implanted ions and crystallizing imperfections.
\end{abstract}

\section{Introduction.}

L'oxygène ajouté aux produits de synthèse de GaAs conduit, sous certaines conditions de préparation, à la formation de GaAs ayant une très grande résistivité [1-4]. L'implantation d'oxygène dans GaAs produit également une couche compensée dans la région implantée [5-6]. Cette technique peut être utilisée pour fabriquer les composants microélectroniques (TEC par exemple) d'où la nécessité de connaitre e mecanisme

compensation qui n'est pas entièrement compris. L'hypothèse d'une interaction oxygène-silicium du substrat a souvent été évoquée pour expliquer la compensation observée. Dans le but de mieux examiner cette interaction, nous avons étudié le phénomène de compensation dans les échantillons de GaAs implantés en oxygène et co-implantés en oxygène et en silicium. Les résultats obtenus sont donnés dans la première partie de cette étude [7].

Par ailleurs, l'implantation ionique dans un semiconducteur $y$ crée de nouveaux défauts qui provien- nent de l'opération d'implantation elle-même ou résultent de la présence d'éléments implantés dans le réseau cristallin du semiconducteur. Dans cette deuxième partie, nous donnons les résultats concernant l'étude des défauts profonds ayant un (ou plusieurs) niveau(x) d'énergie dans la bande interdite des échantillons de GaAs implantés précédemment étudiés.

\section{- océdure de caracterisa $10 n$.}

La préparation des échantillons de GaAs a été décrite dans la première partie lors de l'étude de la compensation. Les tableaux I et II donnent les conditions d'implantation et les caractéristiques des échantillons étudiés.

En ce qui concerne la caractérisation des défauts, nous avons utilisé la méthode DDLTS (DLTS à double corrélation) que nous avons mise au point [8]. Cette méthode permet de déterminer d'une manière précise la signature des défauts et leur 
Tableau I. - Doses et énergies des trois implantations composant la première multi-implantation notée $\Phi_{\mathrm{O}}$. La dose et l'énergie de la première implantation de silicium $\left(\Phi_{\mathrm{Si}_{\mathrm{i}}}\right)$ sont aussi indiquées.

[Doses and energies of oxygen multi-implantation $\left(\Phi_{\mathrm{O}}\right)$ and silicon implantation $\left(\Phi_{\mathrm{Si}_{\mathrm{i}}}\right)$ into GaAs.]

\begin{tabular}{|l|c|c|c|c|}
\hline & \multicolumn{3}{|c|}{ Oxygène $\left(\Phi_{\mathrm{O}}\right)$} & Silicium $\left(\Phi_{\mathrm{Si}}\right)$ \\
\hline dose $\left(\mathrm{cm}^{-2}\right)$ & $1,4 \times 10^{11}$ & $1,05 \times 10^{11}$ & $4,2 \times 10^{10}$ & $10^{11}$ \\
\hline énergie $(\mathrm{keV})$ & 150 & 290 & 550 & 500 \\
\hline
\end{tabular}

Tableau II. - Caractéristiques des échantillons: $\Phi_{\mathrm{O}}$ et $2 \Phi_{\mathrm{O}}$ sont les multi-implantations d'oxygène (les implantations de $2 \Phi_{\mathrm{O}}$ sont effectuées aux mêmes énergies mais à des doses doubles de celles de $\left.\Phi_{\mathrm{O}}\right), \Phi_{\mathrm{Si}}$ et $2 \Phi_{\mathrm{Si}}$ sont les implantations de silicium $\left(2 \Phi_{\mathrm{Si}}\right.$ est une implantation à la même énergie mais à une dose double de celle de $\Phi_{\mathrm{Si}}$ ). Les énergies et les doses de $\Phi_{\mathrm{O}}$ et $\Phi_{\mathrm{Si}}$ sont données dans le tableau I. Le substrat non dopé de GaAs est de type $\mathrm{n}$ avec une concentration électronique de $1,4 \times 10^{16} \mathrm{~cm}^{-3}$. La durée des deux recuits est de $15 \mathrm{~min}$; l'astérisque indique qu'il n'y a pas d'implantation ou de recuit.

[Characteristics of GaAs samples. $\Phi_{\mathrm{O}}$ and $2 \Phi_{\mathrm{O}}$ : oxygen multi-implantation, single and double dose ; $\Phi_{\mathrm{Si}}$ and $2 \Phi_{\mathrm{si}}$ : Si implantation, single and double dose. $\left({ }^{*}\right)$ : no implantation or no annealing. Annealing conditions : $650{ }^{\circ} \mathrm{C}$ or $900{ }^{\circ} \mathrm{C}$ for $15 \mathrm{~min}$.]

\begin{tabular}{|l|c|c|c|c|c|c|c|c|c|}
\hline \multicolumn{1}{|c|}{ Numéro des échantillons } & 1 & 2 & 3 & 4 & 5 & 6 & 7 & 8 & 9 \\
\hline Multi-implantation d'oxygène & $*$ & $*$ & $*$ & $\Phi_{\mathrm{O}}$ & $2 \Phi_{\mathrm{O}}$ & $\Phi_{\mathrm{O}}$ & $2 \Phi_{\mathrm{O}}$ & $\Phi_{\mathrm{O}}$ & $2 \Phi_{\mathrm{O}}$ \\
\hline Implantation de silicium & $*$ & $*$ & $*$ & $*$ & $*$ & $\Phi_{\mathrm{Si}}$ & $*$ & $\Phi_{\mathrm{Si}}$ & $2 \Phi_{\mathrm{Si}}$ \\
\hline Température de recuit $\left({ }^{\circ} \mathrm{C}\right)$ & $*$ & 650 & 900 & 650 & 650 & 650 & 900 & 900 & 900 \\
\hline
\end{tabular}

concentration en fonction de la profondeur. Les mesures ont été effectuées sur des diodes Schottky. Nous rappelons brièvement ici la mise en œuvre de cette méthode et les différentes étapes de mesures. La diode est polarisée en inverse. On superpose à cette polarisation deux impulsions de tension de même durée mais d'amplitudes différentes et décalées d'une «demi-période ». Les signaux transitoires multi-exponentiels produits sont détectés par une détection synchrone qui fournit, à la sortie, deux signaux en phase (a) et en quadrature (b) avec le signal de référence de la détection synchrone.

On enregistre les variations des signaux DDLTS en fonction de la température et on détermine les variations du taux d'émission $e_{\mathrm{n}}$. Les variations de $\log \left(T^{2} / e_{\mathrm{n}}\right)$ en fonction de $1 / T$ permettent de préciser la signature du défaut. Une régression linéaire de l'expression précédente permet d'évaluer $E_{\mathrm{a}}$ et $\sigma_{\infty}$ (énergie d'activation du taux d'émission et section efficace apparente de capture d'un électron). La concentration de chaque type de défaut est déterminée par dérivation numérique de la caractéristique $C(V)$ de la diode de mesure à la tempéra- ture du maximum du pic correspondant dans le spectre DDLTS. Les profils de concentration de défauts en fonction de la profondeur sont déterminés en faisant varier la polarisation inverse de la diode et en maintenant constantes les amplitudes des impulsions de tension.

L'enregistrement des spectres DDLTS $=f(T)$ a été effectué avec une tension de polarisation inverse de $-3 \mathrm{~V}$. Les amplitudes des deux impulsions de tension superposées, à chaque période, à cette polarisation inverse sont respectivement $3 \mathrm{~V}$ et $2 \mathrm{~V}$. L'enregistrement des variations du maximum du signal DDLTS en fonction de la polarisation inverse a été réalisé avec deux valeurs fixes $2,5 \mathrm{eV}$ et $1,5 \mathrm{~V}$ des amplitudes de ces impulsions.

Lorsque sur deux spectres DDLTS de deux échantillons différents, les températures $T_{\mathrm{ma}}$ et $T_{\mathrm{mb}}$ d'un pic sont identiques ou très voisines, on peut alors considérer que les défauts correspondants sont identiques. Pour ne pas surcharger les figures, nous ne représentons que les résultats concernant le signal b (en quadrature avec le signal de référence de la détection synchrone). 


\section{Résultats.}

Les défauts mis en évidence par les spectres DDLTS enregistrés entre 100 et $300 \mathrm{~K}$ sont classés en trois groupes en fonction de la température $T_{\mathrm{mb}}$ des maxima des spectres DDLTS du signal b :

$$
\begin{array}{cl}
T_{\mathrm{mb}}<150 \mathrm{~K} & : \text { défauts type A } \\
150 \mathrm{~K}<T_{\mathrm{mb}}<220 \mathrm{~K} & : \text { défauts type B } \\
T_{\mathrm{mb}}>220 \mathrm{~K} & : \text { défauts type C }
\end{array}
$$

Les résultats déduits des spectres DDLTS sont rassemblés dans le tableau III.

\subsection{Premier groupe De Défauts. $T_{\mathrm{mb}}<150 \mathrm{~K}$.}

Défaut $\mathrm{A}_{5}$. - Ce défaut est présent dans les échantillons de référence $\mathrm{N}^{\circ} 1(*, *, *)$ et $\mathrm{N}^{\circ} 2(*, *$, 650) (Figs. 1a et 1b). Il n'a pu être caractérisé que dans l'échantillon $\mathrm{N}^{\circ} 2$. Sa concentration dans cet échantillon est de l'ordre de $3 \times 10^{14} \mathrm{~cm}^{-3}$ et est indépendante de la profondeur (Fig. 4b). L'énergie d'activation du taux d'émission est $0,14 \mathrm{eV}$ (Tab. III). La signature la plus voisine de ce défaut selon la nomenclature de Martin et al. [9], est EL9 ; cependant l'énergie d'activation de EL9 est plus élevée $(0,22 \mathrm{eV})$.

Défaut $\mathrm{A}_{1}$. - Ce défaut s'observe sur les échantillons $\mathrm{N}^{\circ} 3\left({ }^{*}, *, 900\right), \mathrm{N}^{\circ} 6\left(\Phi_{\mathrm{O}}, \Phi_{\mathrm{si}}, 650\right)$ et $\mathrm{N}^{\circ} 8$ $\left(\Phi_{\mathrm{O}}, \Phi_{\mathrm{Si}}, 900\right)$ (Fig. 1c, Figs. 2c et 3b). Sa concentration dans l'échantillon de référence $\mathrm{N}^{\circ} 3$ est de l'ordre de $2 \times 10^{13} \mathrm{~cm}^{-3}$ (Fig. 4c); elle est de $5 \times 10^{13} \mathrm{~cm}^{-3}$ sur l'échantillon $\mathrm{N}^{\circ} 6$ (Fig. $5 \mathrm{c}$ ) et est trop faible pour être déterminée dans le $\mathrm{N}^{\circ} 8$. L'énergie du taux d'émission est de $0,11 \mathrm{eV}$. Remarquons que cette valeur d'énergie n'apparaît pas souvent dans la littérature. La signature la plus proche de ce défaut, selon la nomenclature de Martin et al. [9] est EL10 (Fig. 7) qui a une énergie d'activation de $0,17 \mathrm{eV}$.

Défaut $\mathrm{A}_{2}$. - Ce défaut est présent sur les échantillons $\mathrm{N}^{\circ} 4\left(\Phi_{\mathrm{O}},{ }^{*}, 650\right), \mathrm{N}^{\circ} 5\left(2 \Phi_{\mathrm{O}}, *, 650\right)$ et $\mathrm{N}^{\circ} 7$ $\left(2 \Phi_{\mathrm{O}}, *, 900\right)$ (Figs. $2 \mathrm{a}$ et $\left.2 \mathrm{~b} ; 3 \mathrm{a}\right)$, qui sont uniquement implantés en oxygène. Les spectres DDLTS des échantillons $\mathrm{N}^{\circ} 4$ et $\mathrm{N}^{\circ} 5$ montrent qu'à ce pic $\mathrm{A}_{2}$ se superpose, du côté des basses températures, un autre pic que nous pensons être le pic $A_{5}$, lequel est mieux distingué sur le spectre de l'échantillon $\mathrm{N}^{\circ} 5$.

Tableau III. - Valeur des températures $T_{\mathrm{ma}}$ et $T_{\mathrm{mb}}$ au maximum des signaux DDLTS $a_{1}$ et $b_{1}$, de l'énergie d'activation $E_{\mathrm{a}}$ du taux d'émission électronique du défaut et de la section efficace de capture apparente $\sigma_{\mathrm{a}}$ de chaque défaut.

\begin{tabular}{|c|c|c|c|c|c|c|c|c|c|}
\hline $\mathrm{N}^{\circ}$ & 1 & 2 & 3 & 4 & 5 & 6 & 7 & 8 & 9 \\
\hline DEF & $\left(A_{5}\right)$ & $\left(A_{5}\right)$ & $\left(A_{1}\right)$ & $\left(A_{2}\right)$ & $\left(\mathrm{A}_{2}\right)$ & $\left(A_{1}\right)$ & $\left(A_{2}\right)$ & $\left(A_{1}\right)$ & $\left(A_{3}\right)$ \\
\hline$T_{\mathrm{ma}}(\mathrm{K})$ & 135 & 135,2 & 123 & 146,4 & 151,5 & 123,8 & 143 & 125,4 & 135 \\
\hline$T_{\mathrm{mb}}(\mathrm{K})$ & 128 & 128,8 & 115,2 & 137,6 & 143,4 & 115,6 & 137,5 & 115,6 & 136 \\
\hline$E_{\mathrm{a}}(\mathrm{eV})$ & & 0,14 & 0,11 & 0,196 & 0,161 & 0,11 & 0,175 & 0,13 & 0,105 \\
\hline$\sigma_{\mathrm{d}}\left(\mathrm{cm}^{2}\right)$ & & $8 \times 10^{-18}$ & $2,5 \times 10^{-18}$ & $5,3 \times 10^{-16}$ & $3 \times 10^{-17}$ & $2 \times 10^{-18}$ & $7,7 \times 10^{-17}$ & $8 \times 10^{-17}$ & $4 \times 10^{-19}$ \\
\hline DEF & $\left(B_{1}\right)$ & $\left(B_{1}\right)$ & $\left(B_{3}\right)$ & $\left(B_{1}\right)$ & $\left(\mathrm{B}_{1}\right)$ & $\left(\mathrm{B}_{2}\right)$ & $\left(B_{2}\right)$ & $\left(B_{2}\right)$ & $\left(B_{2}\right)$ \\
\hline$T_{\mathrm{ma}}(\mathrm{eV})$ & 181,6 & 181,7 & 194,9 & 184,5 & 184,3 & 205,5 & 213,5 & 211,3 & 211 \\
\hline$T_{\mathrm{mb}}(\mathrm{cV})$ & 173,8 & 173,6 & 183 & 177 & 175,1 & 199,1 & 202,9 & 202,1 & 200,5 \\
\hline$E_{\mathrm{a}}(\mathrm{cV})$ & 0,305 & 0,305 & 0,322 & , & , & , & , & , & 3 \\
\hline$\sigma_{\mathrm{a}}\left(\mathrm{cm}^{2}\right)$ & $9 \times 10^{-15}$ & $7,3 \times 10^{-15}$ & $4 \times 10^{-15}$ & $5 \times 10^{-15}$ & $3 \times 10^{-15}$ & $10^{-14}$ & $4 \times 10^{-14}$ & $3 \times 10^{-15}$ & $4 \times 10^{-15}$ \\
\hline DEF & (C) & (C) & & (C) & (C) & (C) & & & \\
\hline$T_{\mathrm{ma}}(\mathrm{K})$ & 280,3 & 280,1 & & 281,4 & 280,1 & 275,3 & & & \\
\hline$T_{\mathrm{mb}}(\mathrm{K})$ & 270,2 & 270,1 & & 271,1 & 270,1 & 265,1 & & & \\
\hline$E_{\mathrm{d}}(\mathrm{cV})$ & 0,574 & 0,563 & & 0,563 & 0,54 & 0,54 & & & \\
\hline$\sigma_{\mathrm{a}}\left(\mathrm{cm}^{2}\right)$ & $3,4 \times 10^{-13}$ & $1,3 \times 10^{-13}$ & & $1,3 \times 10^{-13}$ & $6,4 \times 10^{-14}$ & $6,7 \times 10^{-14}$ & & & \\
\hline
\end{tabular}

[Characteristics of DDLTS peaks in GaAs samples : $T_{\mathrm{ma}}, T_{\mathrm{mb}}$ : peak temperatures ; $E_{\mathrm{a}}$ : apparent activation energy $; \sigma_{\mathrm{a}}$ : apparent capture cross section.] 


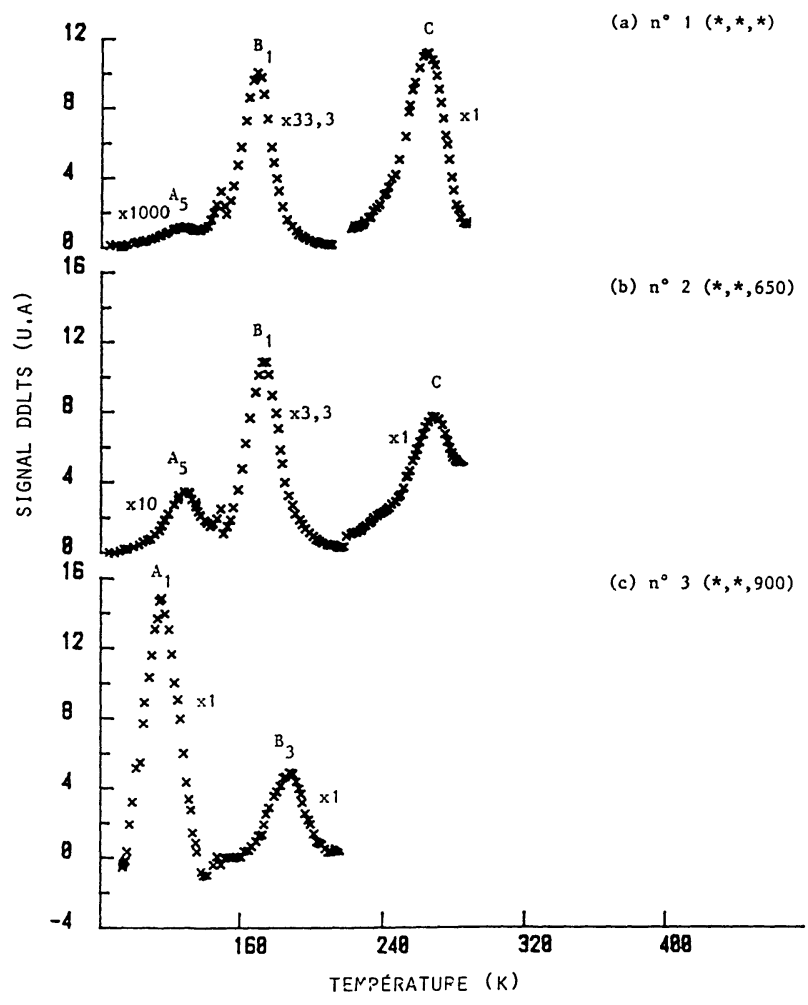

Fig. 1. - Spectre DDLTS des échantillons de GaAs de référence. a : échantillon $\mathrm{N}^{\circ} 1$ non implanté, non recuit ; $\mathrm{b}$ : échantillon $\mathrm{N}^{\circ} 2$ non implanté, recuit à $650^{\circ} \mathrm{C}$ pendant 15 min. c : échantillon $\mathrm{N}^{\circ} 3$ non implanté, recuit à $900^{\circ} \mathrm{C}$ pendant $15 \mathrm{~min}$.

[DDLTS spectra of GaAs substrates. a : unimplanted unannealed GaAs ; b : unimplanted, annealed at $650^{\circ} \mathrm{C}$, $15 \mathrm{~min}$; c : unimplanted, annealed at $900^{\circ} \mathrm{C}, 15 \mathrm{~min}$.]

L'énergie d'activation des taux d'émission du défaut $\mathrm{A}_{2}$ est de l'ordre de $0,16 \mathrm{eV}-0,19 \mathrm{eV}$. La signature EL14 est la plus voisine de celle de ce défaut (Fig. 7). La concentration de ce défaut dépend de la dose d'oxygène; dans la région 0,5 $1,5 \mu \mathrm{m}$, elle passe de $1,5 \times 10^{14} \mathrm{~cm}^{-3}$. $5 \times 10^{14} \mathrm{~cm}^{-3} \quad$ (échantillon $\mathrm{N}^{\circ} 4$ ) à $5 \times 10^{14}$. $1 \times 10^{15} \mathrm{~cm}^{-3}$ (échantillon $\mathrm{N}^{\circ} 5$ qui a reçu une double dose d'oxygène) (Figs. $5 \mathrm{a}$ et $5 \mathrm{~b}$ ). Cette concentration diminue après un recuit à $900^{\circ} \mathrm{C}$ (Fig. 6a). Après ce recuit, il ne reste que le pic $\mathrm{A}_{2}$ dans le spectre DDLTS. Ce défaut est donc moins bien guéri que le défaut $\mathrm{A}_{5}$.

Défaut $\mathrm{A}_{3}$. - Ce défaut est présent dans l'échantillon $\mathrm{N}^{\circ} 9\left(2 \Phi_{\mathrm{O}}, 2 \Phi_{\mathrm{Si}}, 900\right)$ (Fig. 3c). Le spectre DDLTS de cet échantillon montre que le pic $\mathrm{A}_{3}$ est déformé. Il résulte très probablement de la superposition de deux pics : les pics $A_{1}$ et $A_{2}$.

Les résultats obtenus concernant ce premier groupe nous ont amenés à considérer qu'il n'y a en réalité que deux défauts principaux: $\mathrm{A}_{1}$ et $\mathrm{A}_{2}$. $\mathrm{La}$

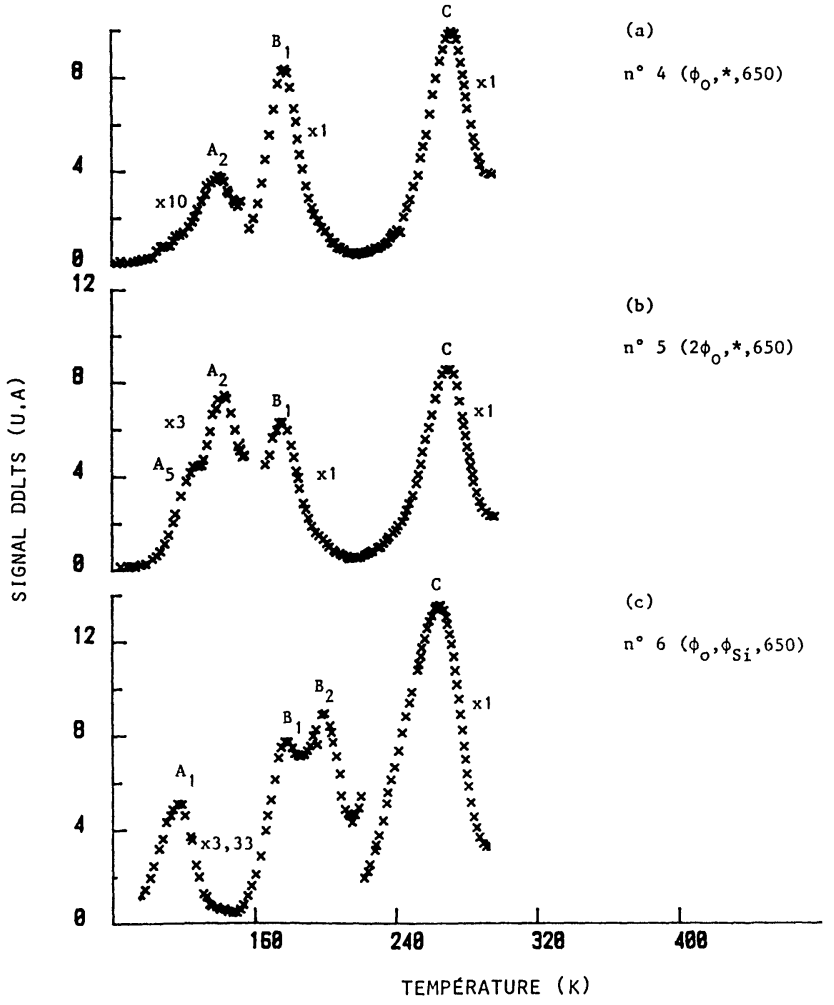

Fig. 2. - Spectre DDLTS des échantillons de GaAs implantés ou co-implantés et recuits à $650^{\circ} \mathrm{C}$. a : échantillon $\mathrm{N}^{\circ} 4$, implanté en oxygène; $\mathrm{b}$ : échantillon $\mathrm{N}^{\circ} 5$, implanté en oxygène, double dose ; $\mathrm{c}$ : échantillon $\mathrm{N}^{\circ} 6$, co-implanté en oxygène et en silicium.

[DDLTS spectra of implanted and annealed $\left(650^{\circ} \mathrm{C}\right.$, 15 min) GaAs. a : sample 4, oxygen implanted GaAs ; b : sample 5, double dose oxygen implanted GaAs ; c : sample 6 , co-implanted (oxygen and $\mathrm{Si}$ ) GaAs.]

superposition des pics correspondant à ces défauts fait apparaître, sur certains échantillons, de nouveaux pics dans les spectres DDLTS (cas des défauts $A_{3}$ et $A_{5}$ ). Le pouvoir séparateur en constante de temps de notre méthode DDLTS ne permet pas de séparer, dans le pic résultant, la contribution de chaque défaut. Dans un tel cas, la signature déduite de ce pic résultant est erronée (cette signature est plus ou moins proche de celle de l'un ou de l'autre des défauts constituants).

\subsection{DEUXIÈME GROUPE DE DÉFAUTS $150 \mathrm{~K}<$ $T_{\mathrm{mb}}<220 \mathrm{~K}$.}

Défaut $\mathrm{B}_{1}$. - Le défaut $\mathrm{B}_{1}$ est mis en évidence dans les échantillons de références $\mathrm{N}^{\circ} 1:\left(^{*},{ }^{*},{ }^{*}\right)$ et $\mathrm{N}^{\circ} 2:(*, *, 650)$ (Figs. 1a et $\left.1 \mathrm{~b}\right)$, mais aussi dans les échantillons $\mathrm{N}^{\circ} 4$ : $\left(\Phi_{\mathrm{O}},{ }^{*}, 650\right), \mathrm{N}^{\circ} 5$ : $\left(2 \Phi_{\mathrm{O}},{ }^{*}\right.$, $650)$ implantés en oxygène et $\mathrm{N}^{\circ} 6:\left(\Phi_{\mathrm{O}}, \Phi_{\mathrm{Si}}, 650\right)$ co-implanté $\mathrm{Si}+\mathrm{O}$, et, recuits à $650^{\circ} \mathrm{C}$ (Figs. 2a, 2b et 2c). Pour ces derniers échantillons on observe une légère diminution de l'énergie d'activation du taux 


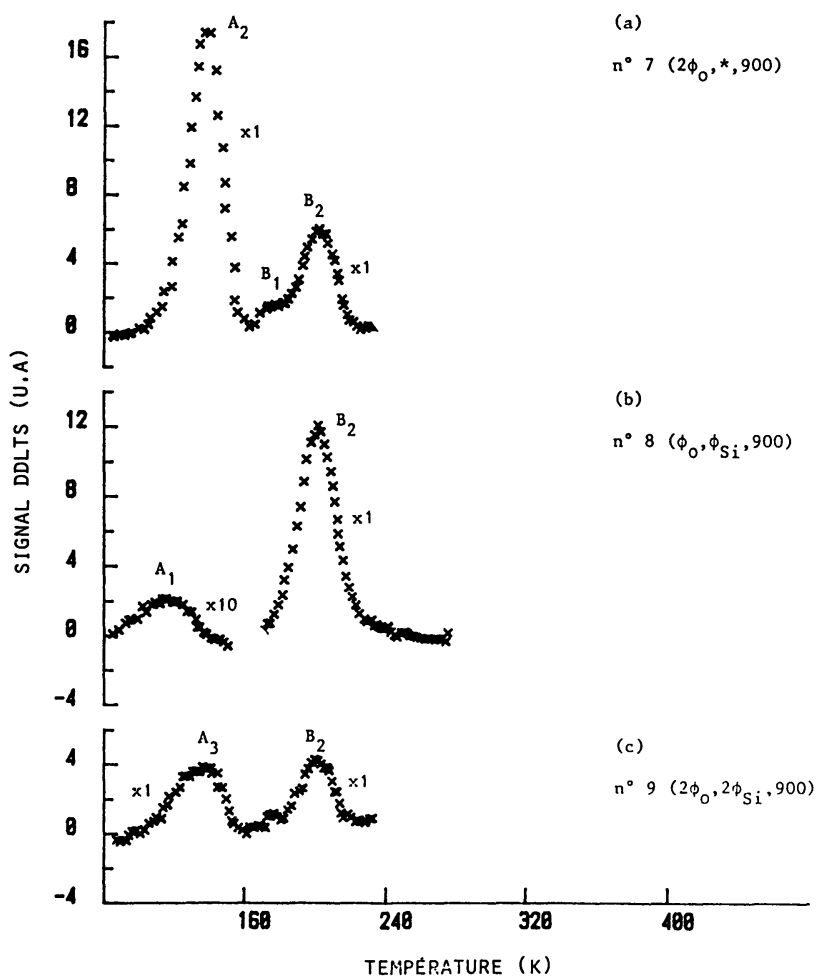

Fig. 3. - Spectre DDLTS des échantillons de GaAs implantés ou co-implantés et recuits à $900^{\circ} \mathrm{C}$. a : échantillon $\mathrm{N}^{\circ} 7$ implanté en oxygène double dose ; $\mathrm{b}$ : échantillon $\mathrm{N}^{\circ} 8$, co-implanté en oxygène et en silicium ; $\mathrm{c}$ : échantillon $\mathrm{N}^{\circ} 9$, co-implanté en oxygène et en silicium, double dose.

[DDLTS spectra of implanted and annealed $\left(900{ }^{\circ} \mathrm{C}\right.$, $15 \mathrm{~min}$ ) GaAs. a : sample 7, double dose oxygen-implanted $\mathrm{GaAs} ; \mathrm{b}$ and $\mathrm{c}$ : samples 8 and 9 , oxygen and $\mathrm{Si}$ co-implanted GaAs.]

d'émission $(0,29$ et $0,28 \mathrm{eV})$ par rapport à celle du taux d'émission des deux premiers échantillons $(0,305 \mathrm{eV})$. La signature du défaut EL7 de la nomenclature de Martin et al. [9] est la plus voisine de celle de $B_{1}$ (Fig. 7).

La concentration de $B_{1}$ est quasi constante et presque identique dans les échantillons non implantés $\mathrm{N}^{\circ} 1$ et $\mathrm{N}^{\circ} 2\left(5-6 \times 10^{14} \mathrm{~cm}^{-3}\right)$ (Figs. $4 \mathrm{a}$ et $\left.4 \mathrm{~b}\right)$ tandis qu'elle augmente légèrement avec la profon-

tés en oxygène et recuits à $650^{\circ} \mathrm{C}$ (Figs. $5 \mathrm{a}$ et $5 \mathrm{~b}$ ).

Défaut $\mathrm{B}_{2}$. - Ce défaut est mis en évidence dans tous les échantillons co-implantés en oxygène et en silicium (recuits à $650^{\circ} \mathrm{C}$ et à $900{ }^{\circ} \mathrm{C}$ ) (échantillons $\mathrm{N}^{\circ} 6,8,9$; Fig. $2 \mathrm{c}$; Figs. $3 \mathrm{~b}$ et $3 \mathrm{c}$ ) et, également dans l'échantillon $\mathrm{N}^{\circ} 7$ multi-implanté en oxygène et recuit à $900^{\circ} \mathrm{C}$. La signature du défaut EL5 est la plus voisine de celle de $B_{2}$. L'énergie d'activation de son taux d'émission est de $\sim 0,35 \mathrm{eV}$.

La concentration de ce défaut augmente avec la profondeur dans le cas de l'échantillon $\mathrm{N}^{\circ} 6\left(\Phi_{\mathrm{O}}\right.$,

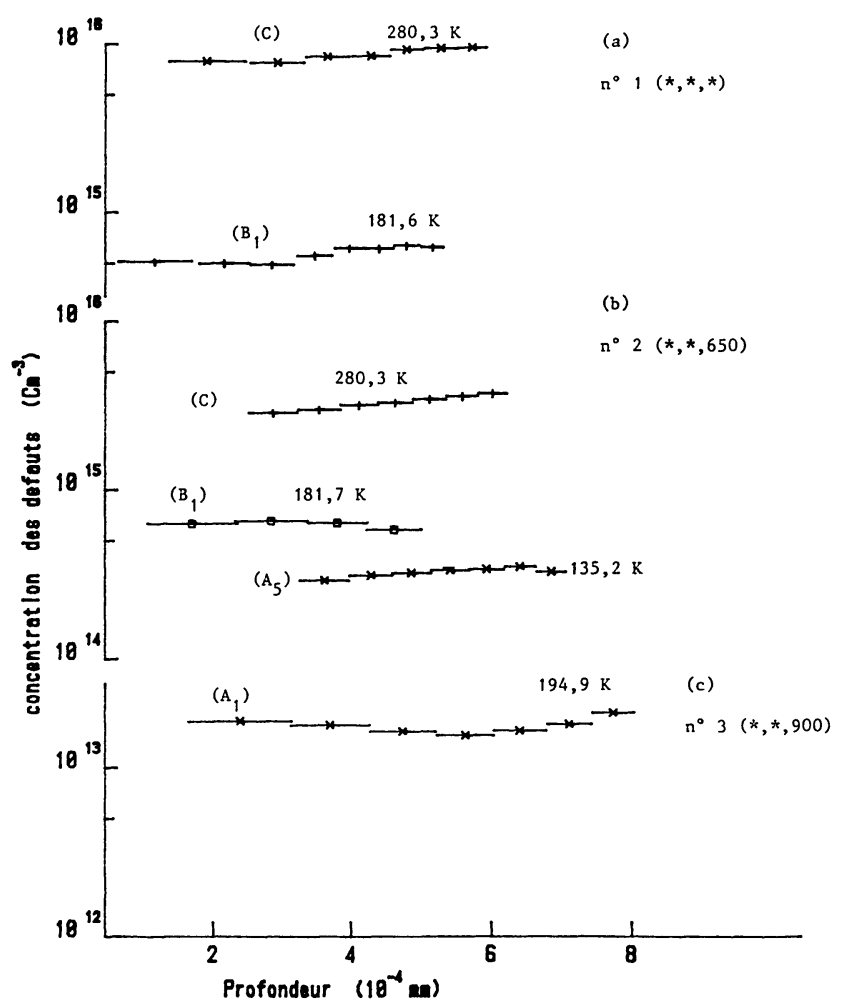

Fig. 4. - Concentration des défauts en fonction de la profondeur des échantillons de $\mathrm{GaAs}$ de référence ( $\mathrm{N}^{\mathrm{os}} 1$, 2 et 3) aux températures des maxima des pics DDLTS.

[Concentration of deep levels at DDLTS maxima pics temperature of unimplanted GaAs substrates. a : unimplanted and unannealed GaAs; $b$ : unimplanted and annealed $\left(650^{\circ} \mathrm{C}, 15 \mathrm{~min}\right) \mathrm{GaAs}$; c : unimplanted and annealed $\left.\left(900^{\circ} \mathrm{C}, 15 \mathrm{~min}\right) \mathrm{GaAs}.\right]$

$\Phi_{\mathrm{Si}}, 650$ ) (co-implanté en oxygène et en silicium et recuit à $\left.650^{\circ} \mathrm{C}\right)(\mathrm{Fig} .5 \mathrm{c})$. Elle est par contre pratiquement indépendante de la profondeur dans l'échantillon $\mathrm{N}^{\circ} 7\left(2 \Phi_{\mathrm{O}}, *, 900\right)$ (implanté seulement en oxygène et recuit à $900^{\circ} \mathrm{C}$ ) (Fig. 6a).

Ce défaut $B_{2}$ co-existe parfois avec le défaut $\mathrm{B}_{1}$ (échantillon $\mathrm{N}^{\circ} 6$, Fig. 2c). Cependant après un recuit à $900{ }^{\circ} \mathrm{C}$, le pic du défaut $\mathrm{B}_{1}$ disparaît pratiquement tandis que celui du défaut $B_{2}$ devient prépondé-

présente un minimum $\left(\sim 10^{13} \mathrm{~cm}^{-3}\right)$ au voisinage de $6 \times 10^{-4} \mathrm{~mm}$ (Fig. $6 \mathrm{~b}$ ). La "guérison " du défaut $B_{1}$ est plus rapide que celle du défaut $B_{2}$ après un recuit à $900^{\circ} \mathrm{C}$.

Défaut $\mathrm{B}_{3}$. - Le défaut $\mathrm{B}_{3}$ est seulement mis en évidence dans l'échantillon de référence recuit à $900{ }^{\circ} \mathrm{C}\left(\mathrm{N}^{\circ} 3:(*, *, 900)\right)$ (Fig. 1c). Les températures $T_{\mathrm{ma}}$ et $T_{\mathrm{mb}}(194,9 ; 183 \mathrm{~K})$ correspondant à ce défaut sont intermédiaires entre celles correspondant aux défauts $B_{1}(181,6 ; 173,8 \mathrm{~K})$ et aux défauts $\mathrm{B}_{2}(211,3 ; 202,3 \mathrm{~K})$. 


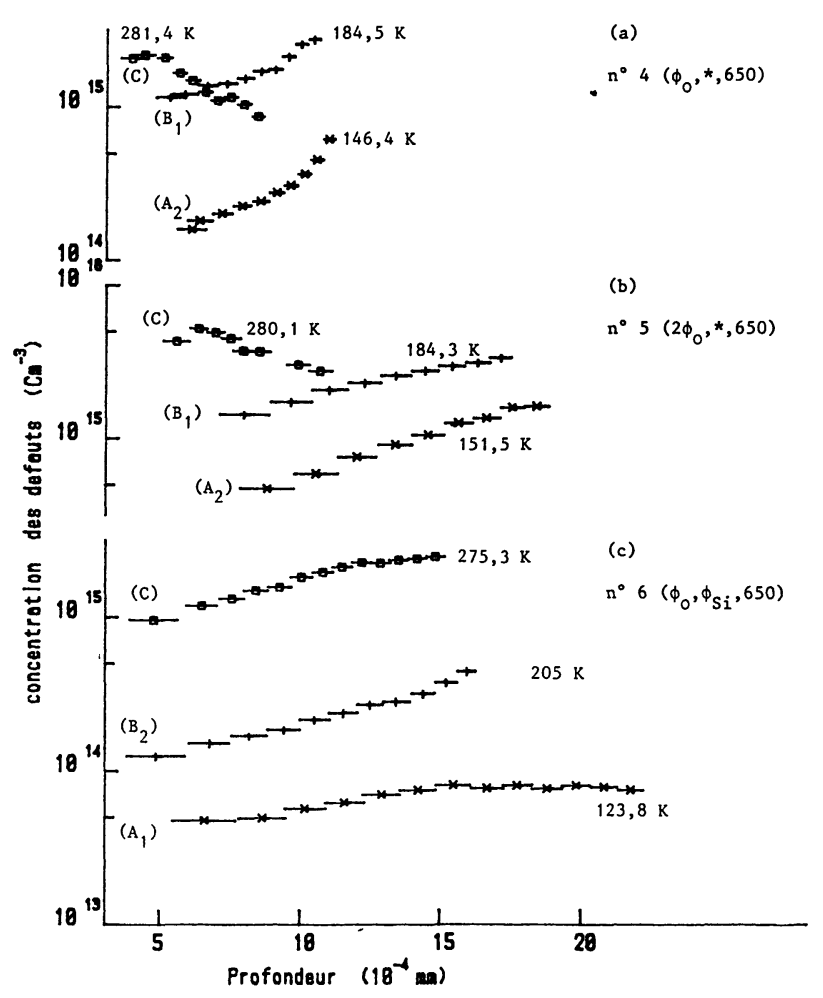

Fig. 5. - Concentration des défauts en fonction de la profondeur des échantillons de GaAs implantés en oxygène (échantillons $\mathrm{N}^{\circ} 4$ et $\mathrm{N}^{\circ} 5$ ) ou co-implantés en oxygène et en silicium (échantillon $\mathrm{N}^{\circ} 6$ ) après recuit à $650^{\circ} \mathrm{C}$ pendant $15 \mathrm{~min}$. et aux températures des maxima des pics DDLTS.

[Concentration of deep-levels at DDLTS maxima pics temperature of oxygen-implanted GaAs (samples 4 and 5) and co-implanted (oxygen and silicium) GaAs (sample 6). Annealed conditions : $650{ }^{\circ} \mathrm{C}, 15 \mathrm{~min}$.]

D'autre part la forme du pic $B_{3}$ dans le spectre DDLTS (Fig. 1c) laisse supposer que ce pic est formé en réalité de la superposition de deux pics : ceux correspondant aux défauts $B_{1}$ et $B_{2}$. L'insuffisance de la sélectivité en constante de temps de la DDLTS ne permet pas de les séparer. Nous ne discuterons pas le cas de ce défaut.

3.3 TROISIÈME GRoupe DE DÉFAUTS. - Ce groupe ne comprend que le défaut $\mathrm{C}$. Il est mis en évidence dans les échantillons de référence $\mathrm{N}^{\circ} 1$ et 2 et dans tous les échantillons recuits à $650^{\circ} \mathrm{C}$ (échantillons $\mathrm{N}^{\circ} 2,4,5$ et 6 ). Par contre, son pic disparaît des spectres DDLTS de tous les échantillons recuits à $900{ }^{\circ} \mathrm{C}$. Ce défaut est donc totalement guéri après ce traitement de recuit.

Les températures $T_{\mathrm{ma}}$ et $T_{\mathrm{mb}}$ (Tab. III) des pics DDLTS de ce défaut coïncident à $1,1 \mathrm{~K}$ près dans tous les échantillons $\mathrm{N}^{\circ} 1,2,4,5$ et 6 . L'énergie d'activation du taux d'émission de ce défaut est de $\sim 0,56 \mathrm{eV}$. La signature de ce défaut est voisine de celle de EL3 selon la nomenclature de Martin et al. [9]. La concentration de ce défaut est pratiquement indépendante de la profondeur dans les échan-

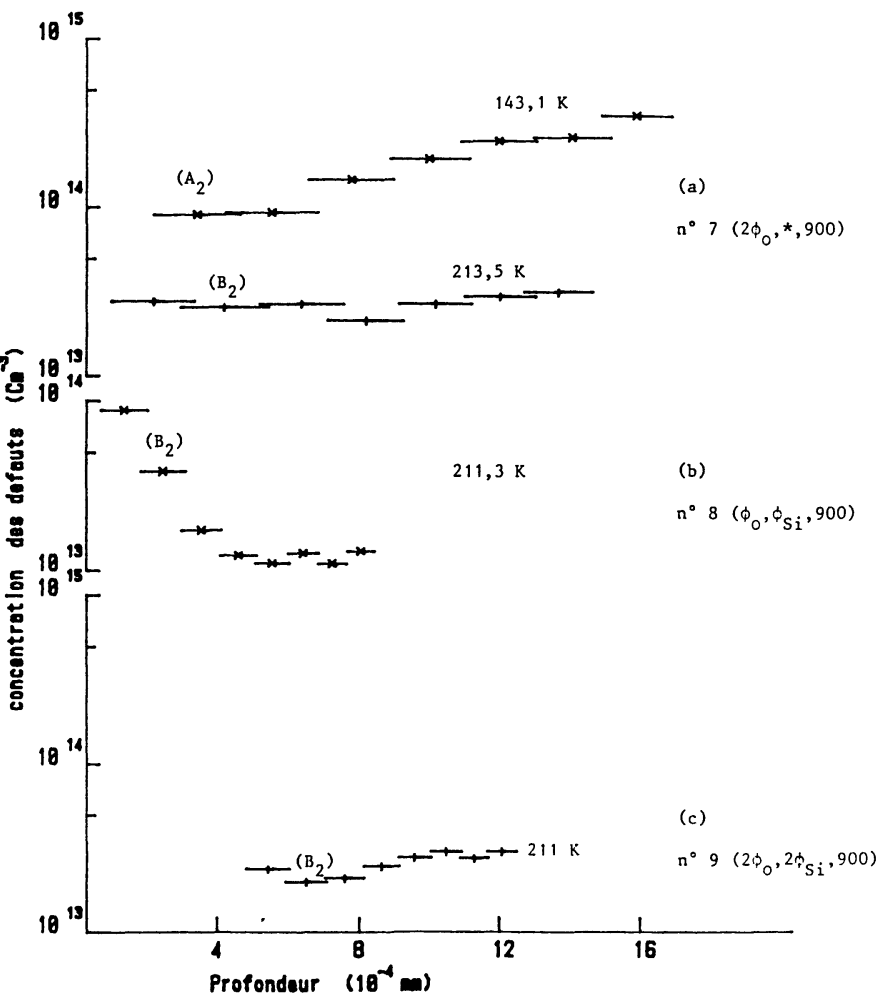

Fig. 6. - Concentration des défauts en fonction de la profondeur des échantillons de GaAs implantés en oxygène (échantillon $\mathrm{N}^{\circ} 7$ ) ou co-implantés en oxygène et en silicium (échantillons $\mathrm{N}^{\circ} 8$ et $\mathrm{N}^{\circ} 9$ ) après recuits à $900^{\circ} \mathrm{C}$ pendant $15 \mathrm{~min}$ et aux températures des maxima des pics DDLTS.

[Concentration at DDLTS maxima pics temperature of deep-levels of oxygen-implanted GaAs (sample 7) and coimplanted (oxygen and $\mathrm{Si}$ ) GaAs (samples 8 and 9). Annealed conditions : $900{ }^{\circ} \mathrm{C}, 15 \mathrm{~min}$.]

tillons de référence $\mathrm{N}^{\circ} 1$ et 2 . Elle est plus faible dans l'échantillon $\mathrm{N}^{\circ} 2$ recuit à $650^{\circ} \mathrm{C}(2 \times$ $\left.10^{15} \mathrm{~cm}^{-3}\right)$ que dans l'échantillon $\mathrm{N}^{\circ} 1$ non recuit $\left(8 \times 10^{15} \mathrm{~cm}^{-3}\right)$. Dans les échantillons $\mathrm{N}^{\circ} 4\left(\Phi_{\mathrm{O}}, *\right.$, $650)$ et $\mathrm{N}^{\circ} 5\left(2 \Phi_{\mathrm{O}},{ }^{*}, 650\right)$ (Figs. $5 \mathrm{a}$ et $\left.5 \mathrm{~b}\right)$ multiimplantés en oxygène seulement, elle présente un maximum vers $0,4-0,6 \mu \mathrm{m}$. La valeur du maximum double lorsque la dose d'oxygène est elle-même doublée. Dans l'échantillon co-implanté en oxygène et en silicium, la concentration de $\mathrm{C}$ augmente avec la profondeur (Fig. 5c).

\subsection{Discussions.}

3.4.1 Premier groupe de défauts: $\mathrm{A}_{1}, \mathrm{~A}_{2}, \mathrm{~A}_{3}$, $\mathrm{A}_{5} 0,10 \mathrm{eV}<E_{\mathrm{a}}<0,20 \mathrm{eV}$. $-\mathrm{A}_{1}$ et $\mathrm{A}_{2}$ et que les défauts $A_{3}$ et $A_{5}$ résultent d'une superposition de $A_{1}$ et de $A_{2}$.

Le défaut $A_{1}$ ne se manifeste que très faiblement dans le spectre DDLTS des échantillons implantés uniquement en oxygène. Par contre, il apparaît très nettement dans les échantillons co-implantés en oxygène et en silicium. Ce défaut semble donc être lié à la co-implantation de ces deux éléments. Sa 


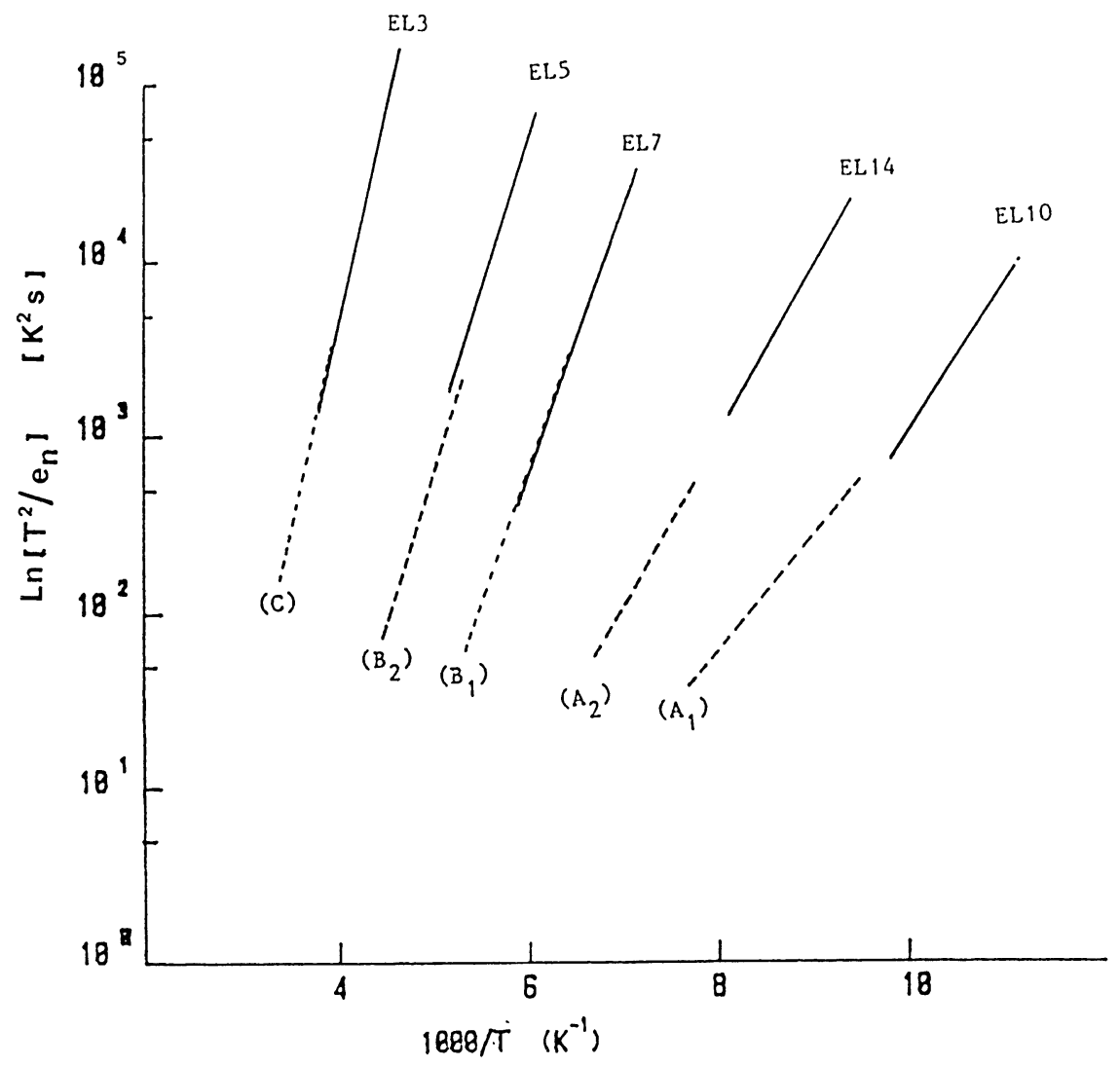

Fig. 7. - Variations de $T^{2} / e_{\mathrm{n}}$ en fonction de $10^{3} / T$. Signatures des défauts mis en évidence dans les échantillons de GaAs de référence, implantés en oxygène et co-implantés en oxygène et en silicium.

[Variations of $T^{2} / e_{\mathrm{n}}$ plotted as a function of $10^{3} / T$ for deep levels of unimplanted GaAs, oxygen implanted and $(\mathrm{O}+\mathrm{Si})$ co-implanted GaAs.]

concentration augmente avec la dose de silicium implanté. Cependant on constate que sa distribution en profondeur n'est pas corrélée à celle du silicium. En effet cet élément diffuse très peu dans GaAs même après un recuit à $900^{\circ} \mathrm{C}[10]$. Nous pensons que $A_{1}$ est un défaut complexe résultant d'une association entre les impuretés dans GaAs et un défaut du réseau cristallin. Sa formation est favorisée par la présence de l'oxygène et du silicium implantés. L'association des impuretés résiduelles et des défauts du réseau cristallin a aussi été suggérée par Look $e t$ $a$. pour exp iquer es e auts ans a $\mathrm{s}$ ec e Bridgman) ayant des énergies d'activation dans le domaine $0,1-0,2 \mathrm{eV}$ qu'ils ont mis en évidence. Cependant le rôle des impuretés silicium et oxygène n'a pas été relevé par ces auteurs. Notons enfin que la présence de $A_{1}$ dans l'échantillon de référence $\mathrm{N}^{\circ} 3$ peut être due à la présence de silicium et d'oxygène résiduels dans le substrat.

Le défaut $\mathrm{A}_{2}\left(E_{\mathrm{a}} \sim 0,16-0,19 \mathrm{eV}\right)$ semble lié à la présence de l'oxygène implanté. En effet, il est présent dans tous les échantillons implantés en oxygène. Le pic DDLTS de ce défaut est particuliè- rement net dans le cas de l'échantillon $\mathrm{N}^{\circ} 7\left(2 \Phi_{\mathrm{O}}\right.$, *, 900) ((Fig. 3a) ; $\left.E_{\mathrm{a}}=0,175 \mathrm{eV}\right)$ ) qui a reçu une double dose d'oxygène. Dans l'échantillon $\mathrm{N}^{\circ} 6$ $\left(\Phi_{\mathrm{O}}, \Phi_{\mathrm{Si}}, 650\right)$ (Fig. 2c) co-implanté en oxygène et en silicium et recuit à $650^{\circ} \mathrm{C}$, ce défaut n'existe plus. Il réapparaît, par contre, faiblement, après un recuit à $900^{\circ} \mathrm{C}$. L'interprétation précise de ce défaut est difficile. Il résulte probablement d'une association complexe entre l'oxygène implanté et les défauts natifs du substrat.

On peut remarquer que la présence de l'oxygène favorise la formation de défauts $\mathrm{A}_{2}$, tandis que celle $\mathrm{du}$ silicium et de l'oxygène favorise la formation de défauts $A_{1}$.

3.4.2 Deuxième groupe de défauts: $\mathrm{B}_{1}, \mathrm{~B}_{2}, \mathrm{~B}_{3}$ $0,28 \mathrm{eV}<E_{\mathrm{a}}<0,39 \mathrm{eV}$.

Défaut $\mathrm{B}_{1}\left(E_{\mathrm{a}} \sim 0,30 \mathrm{eV}\right) .-\mathrm{Ce}$ défaut, détecté dans notre étude sur les échantillons de référence et les échantillons implantés en oxygène, a été également mis en évidence dans les cristaux de GaAs préparés par MBE à $500^{\circ} \mathrm{C}-650^{\circ} \mathrm{C}[9,12,13]$. Blood et al. [12] le considèrent comme lié à une seule 
impureté tandis que Skromm et al. [13] le considèrent comme associé à plusieurs impuretés. Le fait que ce défaut est presque totalement "guéri » après un recuit à $900^{\circ} \mathrm{C}$ aussi bien dans les échantillons de référence que dans les échantillons implantés nous conduit à supposer que ce défaut est un natif et est lié à un désordre du réseau cristallin des échantillons de GaAs. Ce désordre se manifeste dans les cas des échantillons non recuits ou recuits à une température trop basse, et est aggravé par les implantations d'ions. Ce qui explique que la concentration du défaut $B_{1}$ augmente dans les échantillons implantés en oxygène.

Défaut $\mathrm{B}_{2}\left(E_{\mathrm{a}} \sim 0,34-0,39 \mathrm{eV}\right)$. - Rappelons que ce défaut est détecté dans tous les échantillons de GaAs ayant subi un recuit à $900{ }^{\circ} \mathrm{C}$. Sa présence dans l'échantillon de référence non implanté montre qu'il ne résulte pas des dommages causés par les implantations. Son pic apparaît surtout dans le spectre DDLTS des échantillons co-implantés en oxygène et en silicium. Cette observation conduit à penser que $B_{2}$ est peut-être associé à la présence soit du silicium et de l'oxygène.

Des centres profonds de signatures voisines de celle de $\mathrm{B}_{2}$ ont été caractérisés dans des échantillons de GaAs préparés par des méthodes différentes. Henini et al. [15] ont mis en évidence un défaut $\left(E_{\mathrm{a}}=0,35 \mathrm{eV} ; \sigma_{\mathrm{a}}=1,4 \times 10^{-14} \mathrm{~cm}^{-2}\right)$ dans des échantillons de GaAs LEC $(100)$ recuits à $850^{\circ} \mathrm{C}$. Pons et al. [16] ont caractérisé un défaut $P_{1}$ $\left(E_{\mathrm{a}}=0,36 \mathrm{eV} ; \sigma_{\mathrm{a}}=6,9 \times 10^{-15} \mathrm{~cm}^{-2}\right)$ dans des échantillons de GaAs irradiés par des électrons et recuits à $500 \mathrm{~K}$. Ces derniers auteurs ont attribué $\mathrm{P}_{1}$ à un complexe dont fait partie un atome de As interstitiel ou une lacune isolée de As. Cependant, bien que la signature de ces défauts soit voisine de celle de $B_{2}$, nous ne pouvons considérer que la structure de $B_{2}$ soit identique à celle des défauts précédents.

3.4.3 Défaut $\mathrm{C}\left(E_{\mathrm{a}} \sim 0,57 \mathrm{eV}\right)$. - Ce défaut a aussi été mis en évidence dans les cristaux de GaAs préparés par VPE (Vapor Phase Epitaxy) [17] et également dans les échantillons de GaAs implantés en oxygène par Yuba et al. [18] et par Asano et al. [19]. Dans notre cas, ce défaut est présent dans tous les échantillons (échantillons de référence et échantillons implantés et co-implantés) non recuits ou recuits à $650^{\circ} \mathrm{C}$.

Le fait que ce défaut existe sur les échantillons de référence, que sa concentration diminue après un traitement thermique de recuit à $650^{\circ} \mathrm{C}$ et qu'il disparaît après un recuit à $900^{\circ} \mathrm{C}$ conduit à penser que ce défaut, comme le défaut $B_{1}$, est lié au désordre du réseau cristallin de l'échantillon $\mathrm{GaAs}$ de départ. Une implantation d'oxygène suivie d'un recuit provoque une diminution de la concentration de ce défaut au niveau de la couche implantée
(Fig. 5a). La co-implantation en oxygène et en silicium provoque un effet semblable (Fig. 5c). Cette diminution de la concentration du défaut $\mathrm{C}$ dans ces échantillons résulte probablement du réarrangement du réseau cristallin du substrat par suite du recuit à $650^{\circ} \mathrm{C}$. Ce réarrangement n'est complet qu'après un recuit à $900^{\circ} \mathrm{C}$.

Signalons que Martin et al. [20] ont montré que le défaut EL3 voisin du nôtre, qu'ils ont mis en évidence dans le cas du GaAs implanté en oxygène, en bore ou en néon, est un défaut du réseau cristallin produit par l'implantation des ions. Dans le cas de l'implantation en bore [21], Makram-Ebeid et al. ont supposé l'existence d'une bande d'énergie $U$ autour du niveau EL3 pour expliquer le pic très large apparaissant dans les spectres DLTS des échantillons non recuits ou recuits à une température inférieure à $450^{\circ} \mathrm{C}$. Ils considèrent que les défauts associés à cette bande d'énergie $U$ sont liés à l'anti-site $\mathrm{As}_{\mathrm{Ga}}$ [22]. Rappelons que l'anti-site $\mathrm{As}_{\mathrm{Ga}}$ associé à un interstitiel $\mathrm{As}_{\mathrm{i}}$ est maintenant estimé être le défaut EL2 [23, 24].

\section{Conclusions.}

La caractérisation des échantillons de GaAs implantés en oxygène et co-implantés en oxygène et en silicium par notre méthode DDLTS nous a permis de mettre en évidence plusieurs défauts dont l'énergie d'activation du taux d'émission est comprise dans le domaine 0,1-0,6 eV. Ces défauts sont présents en profondeur, au-delà de la région implantée. Leur signature est voisine de celles des défauts mentionnés dans la nomenclature de Martin et al. [9].

Les résultats que nous avons obtenus ne permettent pas de préciser l'origine de ces défauts. Cependant, ils nous amènent à considérer que ces défauts sont tous liés soit au désordre du réseau cristallin, soit aux complexes mettant en jeu à la fois les imperfections du réseau et les impuretés du substrat (impuretés introduites ou résiduelles).

D'autre part, dans aucun échantillon co-implanté en silicium et en oxygène, les variations de la concentration des défauts en fonction de la profondeur ne correspondent à celles de la concentration calculée des éléments implantés. Il en résulte que l'oxygène et le silicium ne sont pas seuls responsables de la présence de défauts dans les cas où ils interviennent. Leur présence dans le substrat semble provoquer une modification des équilibres physicochimiques entre les défauts. Rappelons que ces défauts sont très complexes et que leur interprétation précise est d'autant plus difficile que la faible concentration de l'oxygène et du silicium après implantation, de l'ordre de $10^{15}$ à $10^{16} \mathrm{~cm}^{-3}$, ne peut être évaluée.

Les résultats obtenus semblent confirmer la conclusion que nous avons formulée dans la première 
partie (étude de la compensation) à savoir qu'il y a une certaine interaction entre le silicium et l'oxygène. En effet, si la co-implantation du silicium et de l'oxygène rend négligeable la concentration du défaut $\mathrm{A}_{2}$ (lié à l'implantation d'oxygène), elle favorise par contre la formation des défauts $A_{1}$ et $\mathrm{B}_{2}$.

En ce qui concerne le recuit, un traitement de recuit à haute température $\left(900^{\circ} \mathrm{C}\right)$ est nécessaire pour que la "guérison » des défauts soit suffisamment efficace. Le défaut $B_{1}$ des échantillons coimplantés en oxygène et en silicium est complètement guéri après un tel recuit. Dans le cas des échantillons non soumis à l'implantation un tel traitement est également souhaitable voire indispensable : il permet en effet de supprimer la présence des défauts liés aux désordres cristallins du substrat.

Par ailleurs, nous avons constaté qu'en raison de l'insuffisance de sélectivité en constante de temps, la méthode DDLTS ne permet pas de déterminer les signatures des défauts dont les pics sont voisins dans le spectre DDLTS. Pour résoudre ce problème nous avons mis au point une nouvelle méthode, la méthode FTDLTS (Fourier Transform DLTS) qui sera publiée prochainement.

\section{Bibliographie}

[1] Fertin J. L., Lebailly J. L., Deyris E., Inst. Phys. Conf. Ser. 3, Gallium Arsenide (1967) p. 46.

[2] Woodall J. M., Woods J. F., Solid State Commun. 4 (1966) 33-36.

[3] Woodall J. M., Trans. Metall. Soc. 239 (1967) 378.

[4] Martin G. M., Jacob G., Hallais J. P., Grainger F., Roberts J. A., Clegg B., Blood P., Poiblaud G., J. Phys. C 15 (1982) 1841.

[5] Favennec P. N., Pelous G., Binet M., Baudet $\mathrm{P}$., Ion implantation in semiconductors (Plenum Press) 1974, p. 621.

[6] Favennec P. N., J. Appl. Phys. 47 (1976) 2532.

[7] Partie I: Etude du phénomène de compensation (dans ce même numéro).

[8] Le Bloa A., Dang Tran Quan, Favennec P. N., Revue Phys. Appl. 21 (1986) 489.

[9] Martin G. M., Mitonneau A., Mircea A., Electron. Lett. 13 (1977) 191.

[10] Yed Y. K., Hengehold R. L., Kim Y. Y., EZIS A., Park Y.S., Ehret J. E., J. Appl. Phys. 58 (1985) 4083.

[11] Look D. C., Walters D. C., Meyer J. R., Solid State Commun. 42 (1982) 745.

[12] Blood P., Harris J. J., J. Appl. Phys. 56 (1984) 993.

[13] Shromme B. J., Box S. S., LeE B., Low T. S., LePkowsKi T. R., DeJule R. Y., STIllmaN
[14] Weiner M. E., Jordan A. S., J. Appl. Phys. 43 (1972) 1767.

[15] Henini M., Tuck B., Pauli C. J., Solid State Electr. 29 (1986) 5, 483.

[16] Pons D., Bourgoin J. C., J. Phys. C 18 (1985) 3839.

[17] Mircea A., Mitonneau A., J. Appl. Phys. 8 (1975) 15.

[18] Yuba, Gamo K., Namba S., Proc. 1st Conf. on Ion Beam Modification of Materials (Budapest) Eds. J. Gyulai, T. Lohner and E. Pasztos (1979) 557.

[19] Asano T., Atanassov, Ishiwara H., Furukawa S., Jpn J. Appl. Phys. 20 (1981) 901.

[20] Martin G. M., Terriac P., Makram-Ebeid S., Guillot G., Gavan M., Appl. Phys. Lett. 42 (1983) 61.

[21] Makram-Ebeid S., Gautard D., Devillard P., Martin G. M., Appl. Phys. Lett. 40 (1982) 161.

[22] Martin G. M., Makram-Ebeid S., Physica 116B (1983) 371.

[23] von Bardeleben H. J., Stievenard D., BourgoIn J. C., Appl. Phys. Lett. 47 (1985) 970.

[24] Bourgoin J. C., von Bardeleben H. J., STIEveNARD D., J. Appl. Phys. 64 (1988) R65. 